ISSN 0258-7122

Bangladesh J. Agril. Res. 39(3): 427-435, September 2014

\title{
EFFECT OF OSMOPRIMING ON THE EMERGENCE OF MAIZE (Zea mays L.) SEEDLING
}

\author{
K. U. AHAMMAD ${ }^{1}$, M. M. RAHMAN ${ }^{2}$ AND M. AHMED ${ }^{3}$
}

\begin{abstract}
The research work was carried out at the Seed Laboratory of Agronomy Department of Bangladesh Agricultural University, Mymensingh, Bangladesh during the period from March to November in 2008 to find out the effect of different osmopriming techniques on seedling emergence of maize. Seventeen osmopriming techniques viz., $\mathrm{T}_{1}=1 \% \mathrm{Na}_{2} \mathrm{SO}_{4}, \mathrm{~T}_{2}=3 \% \mathrm{Na}_{2} \mathrm{SO}_{4}, \mathrm{~T}_{3}=5 \%$ $\mathrm{Na}_{2} \mathrm{SO}_{4}, \mathrm{~T}_{4}=1 \% \quad \mathrm{~K}_{2} \mathrm{HPO}_{4}, \quad \mathrm{~T}_{5}=3 \% \quad \mathrm{~K}_{2} \mathrm{HPO}_{4}, \mathrm{~T}_{6}=5 \% \mathrm{~K}_{2} \mathrm{HPO}_{4}, \mathrm{~T}_{7}=1 \%$ $\mathrm{ZnSO}_{4}, \mathrm{~T}_{8}=3 \% \mathrm{ZnSO}_{4}, \mathrm{~T}_{9}=5 \% \mathrm{ZnSO}_{4}, \mathrm{~T}_{10}=1 \% \mathrm{Ca}\left(\mathrm{H}_{2} \mathrm{PO}_{4}\right)_{2}, \mathrm{~T}_{11}=3 \%$ $\mathrm{Ca}\left(\mathrm{H}_{2} \mathrm{PO}_{4}\right)_{2}, \mathrm{~T}_{12}=5 \% \mathrm{Ca}\left(\mathrm{H}_{2} \mathrm{PO}_{4}\right)_{2}, \mathrm{~T}_{13}=1 \% \mathrm{H}_{2} \mathrm{O}_{2}, \mathrm{~T}_{14}=3 \% \mathrm{H}_{2} \mathrm{O}_{2}, \mathrm{~T}_{15}=5 \%$ $\mathrm{H}_{2} \mathrm{O}_{2}, \mathrm{~T}_{16}=$ Hydropriming, $\mathrm{T}_{17}=$ Non-priming (control) were used as experimental variables. Different osmopriming methods on seedling emergence performance of maize was evaluated at two moisture levels viz., 30 and $60 \%$ moisture of saturated sand in the experiment. Germination percentage, germination index and mean germination time were influenced significantly by osmopriming methods. Seed priming with $3 \% \mathrm{ZnSO}_{4}$ showed the highest seedling emergence which was followed by $1 \% \mathrm{H}_{2} \mathrm{O}_{2}$ and $3 \% \mathrm{Ca}\left(\mathrm{H}_{2} \mathrm{PO}_{4}\right)_{2}$.
\end{abstract}

Keywords: Maize, osmopriming, emergence.

\section{Introduction}

Maize is the important cereal crop of Bangladesh. Good seedling establishment of maize is a prerequisite for successful production because it has no capacity to adjust sub-optimal stand by tillering (Finch Savage et al., 2004). Rapid, uniform, and optimum crop stand establishment is necessary for obtaining higher yield of maize. Seed priming can be a simple solution towards expected stand establishment (Harris et al., 2001). Heydecker et al. (1973) defined osmotic seed priming as a pre-sowing treatment in an osmotic solution that allows seeds to imbibe water to proceed to the first stage of germination but prevent radicle protrusion through the seed coat. Seed priming allows the seed to imbibe water slowly, permitting the early stages of germination to begin without radicle protrusion through the seed coat. Osmopriming is the most widely used type of seed priming in which seeds are soaked in aerated low water potential solution (Farooq et al., 2005). Priming of seeds in osmoticums has been reported to be an

\footnotetext{
${ }^{1}$ Senior Scientific Officer, RARS, Bangladesh Agricultural Research Institute (BARI), Jessore, ${ }^{2 \& 3}$ Professor, Department of Agronomy, Bangladesh Agricultural University (BAU), Mymansingh, Bangladesh.
} 
economical, simple and a safe technique for seedling establishment and crop production under stressed conditions (Guzman and Olave, 2006).

Different osmotica can be used in seed priming, such as osmotica used include Polyethylene Glycol ( $\mathrm{PEG}$ ), $\mathrm{KNO}_{3}, \mathrm{~K}_{3} \mathrm{PO}_{4}, \mathrm{KH}_{2} \mathrm{PO}_{4}, \mathrm{MgSO}_{4}, \mathrm{NaCl}$, manitol and others (Lee and Kim, 1999; Basra et al., 2005). Sunflower seeds treated with PEG-8000 solution at $15{ }^{\circ} \mathrm{C}$ had an increased germination rate (Bailly et al., 2000). Kattimani et al. (1999) found that seed primed with nitrate solutions produced more vigorous seedlings, higher dry matter and root length. The beneficial effect of osmopriming on germination has been reported in tomato seeds (Ozbingol et al., 1998. Guzman and Olave (2006) reported that seed priming with nitrate solutions resulted in an improved germination rate, radicle growth, and germination index. It has been observed that physiological and biochemical changes take place during the seed treatments which could allow seeds to begin the germination sequences before sowing (Basra et al., 2005; Ghiyasi et al., 2008).

Although quite a good number of works have been done on seed priming of maize in abroad but under Bangladesh condition such works are a few. To use the benefit of seed priming technology in Bangladesh, it is necessary to study the effects of seed priming in plant stand establishment of maize. The present study was therefore, undertaken to study the effect of different osmopriming techniques on seedling emergence of maize.

\section{Materials and Method}

The research was conducted at the Seed Laboratory of Agronomy Department of Bangladesh Agricultural University, Mymensingh during the period from March to November in 2008. Seventeen osmopriming treatments were $\mathrm{T}_{1}=1 \% \mathrm{Na}_{2} \mathrm{SO}_{4}$, $\mathrm{T}_{2}=3 \% \mathrm{Na}_{2} \mathrm{SO}_{4}, \mathrm{~T}_{3}=5 \% \mathrm{Na}_{2} \mathrm{SO}_{4}, \mathrm{~T}_{4}=1 \% \mathrm{~K}_{2} \mathrm{HPO}_{4}, \mathrm{~T}_{5}=3 \% \mathrm{~K}_{2} \mathrm{HPO}_{4}, \mathrm{~T}_{6}=5 \%$ $\mathrm{K}_{2} \mathrm{HPO}_{4}, \mathrm{~T}_{7}=1 \% \mathrm{ZnSO}_{4}, \mathrm{~T}_{8}=3 \% \mathrm{ZnSO}_{4}, \mathrm{~T}_{9}=5 \% \mathrm{ZnSO}_{4}, \mathrm{~T}_{10}=1 \% \mathrm{Ca}\left(\mathrm{H}_{2} \mathrm{PO}_{4}\right)_{2}$, $\mathrm{T}_{11}=3 \% \mathrm{Ca}\left(\mathrm{H}_{2} \mathrm{PO}_{4}\right)_{2}, \mathrm{~T}_{12}=5 \% \mathrm{Ca}\left(\mathrm{H}_{2} \mathrm{PO}_{4}\right)_{2}, \mathrm{~T}_{13}=1 \% \mathrm{H}_{2} \mathrm{O}_{2}, \mathrm{~T}_{14}=3 \% \mathrm{H}_{2} \mathrm{O}_{2}, \mathrm{~T}_{15}$ $=5 \% \quad \mathrm{H}_{2} \mathrm{O}_{2}, \mathrm{~T}_{16}=$ Hydropriming, and $\mathrm{T}_{17}=$ Non-priming (control). The experiment was laid out in completely randomized design with three replications. For each of these chemicals 1,3 , and 5\% solutions were prepared by adding 2, 6 , and $10 \mathrm{~g}$ chemicals, respectively, in $200 \mathrm{ml}$ distilled water. For $\mathrm{H}_{2} \mathrm{O}_{2}, 1,3$. and $5 \%$ solution were created by adding 2,6 , and $10 \mathrm{ml} \mathrm{H}_{2} \mathrm{O}_{2}$ in 198,194 , and $190 \mathrm{ml}$ water, respectively. Three hundred seeds of maize (var. BARI Hybrid Maize-5) were taken in plastic bowl and were dipped in the osmotic solution as per experimental specification. After 18 hours of soaking, the seeds were taken out from the bowl and washed under tap water for several times. Then it was surface dried for two hours under shade. Hydropriming was done by dipping 300 seeds in 
$200 \mathrm{ml}$ water. Plastic pots $(21 \mathrm{~cm}$ diameter and $9 \mathrm{~cm}$ depth) were filled with 2.5 $\mathrm{kg}$ dried sand. Two levels of moisture viz., $30 \%$ and $60 \%$ moisture of the saturated sand was created in the pot sand by adding 195 and $390 \mathrm{ml}$ distilled water, respectively. Fifty seeds were placed in the sand at $2 \mathrm{~cm}$ depth maintaining equal distance from seed to seed on 11 March 2008. The seedling emergence performance was tested at 30 and $60 \%$ moisture of saturated sand. After seed placement in the pot no water was added in the sand until completion of the experiment. The trial was repeated twice on 20 October and 10 November 2008. Data on germination percentage, germination index, and mean germination time were recorded as follows:

i. Germination percentage: The seedlings were counted daily until complete emergence. Germination was calculated in percentage using the following formula:

$\%$ Germination $=\frac{\text { Number of seeds germinated }}{\text { Number of seeds sown }} \times 100$

ii. Germination index: The germination index (GI) was calculated by following formula (AOSA, 1983):

$\mathrm{GI}=\frac{\text { Number of germinated seeds }}{\text { Day sof first count }}+--------\frac{\text { Number of germinated seed }}{\text { Day sof final count }}$

iii. Mean germination time (MGT): The mean germination time (days) was calculated according to the following formula (Scott et al., 1984):

$\operatorname{MGT}($ days $)=\frac{? \mathrm{Ti} \mathrm{Ni}}{\mathrm{S}}$

Where, $\mathrm{Ti}=$ Number of days after beginning of experiment

$\mathrm{Ni}=$ Number of seeds germinated on day $\mathrm{i}$

$\mathrm{S}=$ Total number of seeds germinated

Data were subjected to statistical analysis using ANOVA technique through computer based statistical package programme MSTATC. Arc sine transformations were done for percentage data where applicable before the analysis (Gomez and Gomez, 1984). Mean comparison was done by Duncun's Multiple Range Test (DMRT).

\section{Results}

\section{Germination percentage}

Germination percentage of maize seed varied significantly due to different osmopriming treatments both at 30 and $60 \%$ moisture levels (Table 1). In all the 
three trials, seeds primed with $3 \% \quad \mathrm{ZnSO}_{4}$ solution showed the highest germination, which was followed by the seeds primed with $1 \% \mathrm{H}_{2} \mathrm{O}_{2}, 3 \% \mathrm{Ca}$ $\left(\mathrm{H}_{2} \mathrm{PO}_{4}\right)_{2}$ and the lowest was found with control. Hydropriming performed better than some of the chemical priming but gave much lower germination than those primed with $3 \% \mathrm{ZnSO}_{4}, 1 \% \mathrm{H}_{2} \mathrm{O}_{2}$, and $3 \% \mathrm{Ca}\left(\mathrm{H}_{2} \mathrm{PO}_{4}\right)_{2}$ solution. However, any of the chemical priming treatment was superior to non-priming. At $30 \%$ moisture level, mean germination was highest $\left((75 \%)\right.$ for primed with $3 \% \mathrm{ZnSO}_{4}$ and it was lowest (47\%) at non-priming. Similarly, at $60 \%$ moisture, mean germination was the highest $(85 \%)$ for priming with $3 \% \mathrm{ZnSO}_{4}$ and it was lowest $(63 \%)$ from non-primed control.

\section{Germination index}

Germination index of maize seed also varied significantly due to different osmopriming treatments when tested both at 30\% and 60\% moisture levels (Table 2). In all the three trials, seed priming with $3 \% \mathrm{ZnSO}_{4}$ showed the highest germination index, which was followed by seed primed with $1 \% \mathrm{H}_{2} \mathrm{O}_{2}$ and $3 \%$ $\mathrm{Ca}\left(\mathrm{H}_{2} \mathrm{PO}_{4}\right)_{2}$. The lowest germination index was found with non-priming. Hydropriming performed better than some of the chemical primings but gave much lower germination index than $3 \% \mathrm{ZnSO}_{4}, 1 \% \mathrm{H}_{2} \mathrm{O}_{2}$, and $3 \% \mathrm{Ca}\left(\mathrm{H}_{2} \mathrm{PO}_{4}\right)_{2}$. However, any of the chemical priming treatment was superior to non-priming control. At 30\% moisture level, the mean germination index was highest ((22.17) with $3 \% \mathrm{ZnSO}_{4}$ and it was lowest (11.00) from non-priming. At $60 \%$ moisturethe mean germination index was highest (25.74) with $3 \% \mathrm{ZnSO} 4$ and it was lowest (15.62) from non priming control.

\section{Mean germination time}

Mean germination time of maize seed varied significantly due to different osmopriming treatments for tests both at 30 and $60 \%$ moisture levels (Table 3). In the all trials, priming with $3 \% \mathrm{ZnSO}_{4}$ showed the lowest mean germination time, which was followed by $1 \% \mathrm{H}_{2} \mathrm{O}_{2}, 3 \% \mathrm{Ca}\left(\mathrm{H}_{2} \mathrm{PO}_{4}\right)_{2}$ and the highest was found with non-priming control. Hydropriming performed better than some of the chemical priming but gave higher mean germination time than $3 \% \mathrm{ZnSO}_{4}$, $1 \% \mathrm{H}_{2} \mathrm{O}_{2}$, and $3 \% \mathrm{Ca}\left(\mathrm{H}_{2} \mathrm{PO}_{4}\right)_{2}$. However, any of the chemical priming treatment was superior to non-priming control. At 30\% moisture level, the average mean germination time was lowest ((3.40 day) with $3 \% \mathrm{ZnSO}_{4}$ and it was highest (4.54 day) in control treatment. Similarly, at $60 \%$ moisture level, the average mean germination time was lowest (3.39 day) for seeds primed with $3 \% \mathrm{ZnSO}_{4}$ and it was the highest (4.52 day) for non priming control. 
Table 1. Effect of osmopriming treatment on germination percentage of maize seed during 2008.

\begin{tabular}{|c|c|c|c|c|c|c|c|c|}
\hline \multirow{3}{*}{ Treatments } & \multicolumn{8}{|c|}{ Germination percentage } \\
\hline & \multicolumn{4}{|c|}{$30 \%$ moisture } & \multicolumn{4}{|c|}{$60 \%$ moisture } \\
\hline & Trial-1(March) & Trial-2 (Oct.) & Trial-3 (Nov.) & Mean & Trial-1(March) & Trial-2 (Oct.) & Trial-3(Nov.) & Mean \\
\hline $1 \% \mathrm{Na}_{2} \mathrm{SO}_{4}$ & $63(50.49) \mathrm{cd}$ & $68(53.59) \mathrm{c}$ & $60(48.99) \mathrm{e}-\mathrm{g}$ & $63(51.02) \mathrm{de}$ & $72(56.01) \mathrm{cd}$ & $78(59.85) \mathrm{cd}$ & $78(59.85) \mathrm{de}$ & $76(58.57) \mathrm{d}-\mathrm{f}$ \\
\hline $3 \% \mathrm{Na}_{2} \mathrm{SO}_{4}$ & $59(48.23) \mathrm{e}$ & $65(51.64) \mathrm{d}$ & $56(46.75) f-h$ & $60(48.87) f$ & $68(53.59) \mathrm{d}-\mathrm{f}$ & $74(57.26) \mathrm{de}$ & 74 (57.26)ef & $72(56.04) h-j$ \\
\hline $5 \% \mathrm{Na}_{2} \mathrm{SO}_{4}$ & $54(45.63) \mathrm{f}$ & $60(48.98) \mathrm{e}$ & $51(44.15) \mathrm{hi}$ & $55(46.25) \mathrm{g}$ & 65 (51.64)fg & 71 (55.19)ef & $71(55.61) f g$ & $69(54.15) \mathrm{jk}$ \\
\hline $1 \% \mathrm{~K}_{2} \mathrm{HPO}_{4}$ & $59(48.23) \mathrm{e}$ & $65(51.64) \mathrm{d}$ & $57(47.11) f-h$ & $60(48.99) f$ & $71(55.610 \mathrm{c}-\mathrm{e}$ & $77(59.43) \mathrm{cd}$ & 78 (59.85)de & $76(58.29) \mathrm{d}-\mathrm{g}$ \\
\hline $3 \% \mathrm{~K}_{2} \mathrm{HPO}_{4}$ & $51(44.15) \mathrm{f}$ & $57(47.49) \mathrm{e}$ & $53(45.26) \mathrm{g}-\mathrm{i}$ & $54(45.63) \mathrm{g}$ & 65 (51.64)fg & 71 (55.19)ef & 74 (57.26)ef & $70(54.70) \mathrm{i}-\mathrm{k}$ \\
\hline $5 \% \mathrm{~K}_{2} \mathrm{HPO}_{4}$ & $45(40.46) \mathrm{g}$ & $51(43.78) \mathrm{f}$ & $47(41.56) \mathrm{i}$ & $49(42.67) \mathrm{h}$ & $63(50.50) \mathrm{g}$ & $69(54.01) \mathrm{f}$ & $68(53.60) \mathrm{g}$ & $66(52.71) \mathrm{k}$ \\
\hline $1 \% \mathrm{ZnSO}_{4}$ & $63(50.87) \mathrm{cd}$ & $68(53.59) \mathrm{c}$ & $72(56.03) \mathrm{a}-\mathrm{c}$ & $68(53.50) b c$ & $74(57.26) \mathrm{bc}$ & $80(61.21) b c$ & $83(63.64) b c$ & $79(60.70) b c$ \\
\hline $3 \% \mathrm{ZnSO}_{4}$ & $73(56.42) \mathrm{a}$ & $74(57.25) \mathrm{a}$ & $77(59.43) \mathrm{a}$ & $75(57.69) \mathrm{a}$ & $81(61.67) \mathrm{a}$ & $86(65.66) \mathrm{a}$ & 87 (66.79)a & $85(64.70) \mathrm{a}$ \\
\hline $5 \% \mathrm{ZnSO}_{4}$ & $63(50.87) \mathrm{cd}$ & $69(54.38) \mathrm{bc}$ & $69(54.00) \mathrm{b}-\mathrm{d}$ & $67(53.09) \mathrm{b}-\mathrm{d}$ & $71(55.59) \mathrm{c}-\mathrm{e}$ & $77(59.40) \mathrm{cd}$ & $79(60.76) \mathrm{cd}$ & 76 (58.59) d-f \\
\hline $1 \% \mathrm{Ca}\left(\mathrm{H}_{2} \mathrm{PO}_{4}\right)_{2}$ & 61 (49.73)de & $67(53.20) \mathrm{cd}$ & 69 (54.39)b-d & $66(52.44) \mathrm{cd}$ & 68 (53.59)d-f & 74 (57.26)de & 79 (60.77)cd & $74(57.21) \mathrm{f}-\mathrm{h}$ \\
\hline $3 \% \mathrm{Ca}\left(\mathrm{H}_{2} \mathrm{PO}_{4}\right)_{2}$ & $65(52.02) \mathrm{bc}$ & 71 (55.59)ab & 73 (56.85)ab & $70(54.83) \mathrm{b}$ & $76(58.31) \mathrm{b}$ & $83(63.12) b$ & 85 (64.59)ab & $81(62.23) b$ \\
\hline $5 \% \mathrm{Ca}\left(\mathrm{H}_{2} \mathrm{PO}_{4}\right)_{2}$ & 61 (49.74)de & $67(53.20) \mathrm{cd}$ & $63(50.54) d-f$ & 64 (51.16)de & 67 (53.21)ef & 72 (56.01)ef & $78(59.85) \mathrm{de}$ & $72(56.36) \mathrm{g}-\mathrm{I}$ \\
\hline $1 \% \mathrm{H}_{2} \mathrm{O}_{2}$ & $67(52.80) \mathrm{b}$ & 71 (55.59)ab & $73(56.83) \mathrm{ab}$ & $70(55.08) \mathrm{b}$ & $77(58.98) b$ & $84(63.83) b$ & 85 (65.11)ab & $82(62.53) b$ \\
\hline $3 \% \mathrm{H}_{2} \mathrm{O}_{2}$ & $64(51.25) b-d$ & $69(54.38) b c$ & $70(54.78) \mathrm{bc}$ & $68(53.48) b c$ & $73(56.83) b c$ & $79(60.750 \mathrm{bc}$ & $81(61.67) \mathrm{cd}$ & $78(59.75)$ c-e \\
\hline $5 \% \mathrm{H}_{2} \mathrm{O}_{2}$ & $61(49.73) \mathrm{de}$ & $67(53.19) \mathrm{cd}$ & $66(52.42) c-e$ & $65(51.78) \mathrm{cd}$ & $72(56.03) \mathrm{cd}$ & $78(59.88) \mathrm{cd}$ & 75 (57.67)ef & 75 (57.86) e-h \\
\hline Hydropriming & $59(48.23) \mathrm{e}$ & $64(51.26) \mathrm{d}$ & 60 (48.99)e-g & 61 (49.49)ef & $74(57.26) b c$ & $80(61.21) b c$ & $81(61.71) \mathrm{cd}$ & $78(60.06) \mathrm{cd}$ \\
\hline Non-priming & $43(39.71) \mathrm{g}$ & 49 (43.04)f & $53(45.26) \mathrm{g}-\mathrm{i}$ & $47(41.93) \mathrm{h}$ & $57(47.48) \mathrm{h}$ & $63(50.88) \mathrm{g}$ & $68(53.59) \mathrm{g}$ & $63(50.66) 1$ \\
\hline $\mathrm{S}_{\mathrm{x}}^{-}$ & 0.575 & 0.601 & 1.255 & 0.653 & 0.822 & 0.878 & 0.921 & 0.676 \\
\hline Level of signific. & $* *$ & $* *$ & $* *$ & $* *$ & $* *$ & $* *$ & $* *$ & $* *$ \\
\hline CV (\%) & 2.04 & $2.79 \quad 2.01$ & 4.28 & 2.24 & 2.59 & 2.59 & 2.66 & 2.02 \\
\hline
\end{tabular}

Figures in parentheses are arc sine transformed values of germination percentage.

Figures with similar letter(s) or without letter within the column do not differ significantly whereas figures with dissimilar letter(s) differ significantly at 0.05 .

level of probability by DMRT, $* *=$ Significant at 0.01 level of probability. 
Table 2. Effect of osmopriming treatment on germination index of maize seed during 2008.

\begin{tabular}{|c|c|c|c|c|c|c|c|c|}
\hline \multirow{3}{*}{ Treatments } & \multicolumn{8}{|c|}{ Germination index } \\
\hline & \multicolumn{4}{|c|}{$30 \%$ moisture } & \multicolumn{4}{|c|}{$60 \%$ moisture } \\
\hline & Trial-1(March) & Trial-2 (Oct.) & Trial-3(Nov.) & Mean & $\begin{array}{c}\text { Trial- } \\
\text { 1(March) }\end{array}$ & Trial-2 (Oct.) & $\begin{array}{c}\text { Trial- } \\
\text { 3(Nov.) }\end{array}$ & Mean \\
\hline $1 \% \mathrm{Na}_{2} \mathrm{SO}_{4}$ & $19.08 c-e$ & $20.71 c-e$ & $15.30 \mathrm{gh}$ & $18.36 \mathrm{e}-\mathrm{g}$ & $22.02 \mathrm{~cd}$ & $23.78 d-f$ & $21.34 \mathrm{de}$ & $22.38 \mathrm{ef}$ \\
\hline $3 \% \mathrm{Na}_{2} \mathrm{SO}_{4}$ & $16.86 \mathrm{~g}$ & $18.70 \mathrm{~g}$ & $13.27 \mathrm{ij}$ & $16.27 \mathrm{~h}$ & $20.02 \mathrm{fg}$ & 22.03hi & $19.71 \mathrm{f}$ & 20.59hi \\
\hline $5 \% \mathrm{Na}_{2} \mathrm{SO}_{4}$ & $15.25 \mathrm{~h}$ & $17.09 \mathrm{~h}$ & $11.98 \mathrm{j}$ & $14.77 \mathrm{i}$ & $18.25 \mathrm{hi}$ & $19.98 \mathrm{j}$ & $17.91 \mathrm{~g}$ & $18.72 \mathrm{j}$ \\
\hline $1 \% \mathrm{~K}_{2} \mathrm{HPO}_{4}$ & $17.60 \mathrm{fg}$ & $19.33 \mathrm{fg}$ & $15.66 \mathrm{f}-\mathrm{h}$ & $17.53 \mathrm{~g}$ & $21.98 \mathrm{~cd}$ & $23.65 \mathrm{~d}-\mathrm{g}$ & $21.83 \mathrm{~cd}$ & $22.49 \mathrm{ef}$ \\
\hline $3 \% \mathrm{~K}_{2} \mathrm{HPO}_{4}$ & $15.20 \mathrm{~h}$ & $17.01 \mathrm{~h}$ & $14.25 \mathrm{hi}$ & $15.49 \mathrm{hi}$ & $19.22 \mathrm{gh}$ & $21.06 \mathrm{i}$ & $20.07 \mathrm{ef}$ & $20.12 \mathrm{i}$ \\
\hline $5 \% \mathrm{~K}_{2} \mathrm{HPO}_{4}$ & $12.56 \mathrm{i}$ & $14.40 \mathrm{i}$ & $12.09 \mathrm{j}$ & $13.02 \mathrm{j}$ & $17.33 \mathrm{i}$ & $19.01 \mathrm{j}$ & $17.83 \mathrm{~g}$ & $18.06 \mathrm{j}$ \\
\hline $1 \% \mathrm{ZnSO}_{4}$ & $19.26 \mathrm{~b}-\mathrm{d}$ & 20.71c-e & $19.39 \mathrm{~cd}$ & $19.79 \mathrm{~cd}$ & $22.41 b c$ & $24.25 \mathrm{c}-\mathrm{e}$ & $23.06 \mathrm{bc}$ & $23.24 \mathrm{de}$ \\
\hline $3 \% \mathrm{ZnSO}_{4}$ & $21.31 \mathrm{a}$ & $22.89 \mathrm{a}$ & $22.32 \mathrm{a}$ & $22.17 \mathrm{a}$ & $25.09 \mathrm{a}$ & $26.79 \mathrm{a}$ & $25.34 \mathrm{a}$ & $25.74 a$ \\
\hline $5 \% \mathrm{ZnSO}_{4}$ & $18.99 \mathrm{c}-\mathrm{e}$ & $20.74 c-e$ & $17.42 \mathrm{ef}$ & $19.05 \mathrm{~d}-\mathrm{f}$ & $21.64 c-e$ & $23.28 \mathrm{e}-\mathrm{g}$ & $21.82 \mathrm{~cd}$ & $22.25 f$ \\
\hline $1 \% \mathrm{Ca}\left(\mathrm{H}_{2} \mathrm{PO}_{4}\right)_{2}$ & $18.45 \mathrm{~d}-\mathrm{f}$ & $20.25 d-f$ & $19.24 d$ & $19.31 \mathrm{c}-\mathrm{e}$ & $20.62 \mathrm{ef}$ & $22.55 \mathrm{gh}$ & $21.99 \mathrm{~cd}$ & $21.72 \mathrm{fg}$ \\
\hline $3 \% \mathrm{Ca}\left(\mathrm{H}_{2} \mathrm{PO}_{4}\right)_{2}$ & $19.96 b c$ & $21.71 b c$ & $21.09 \mathrm{a}-\mathrm{c}$ & $20.92 b$ & $23.42 b$ & $25.29 b c$ & $24.08 \mathrm{ab}$ & $24.26 \mathrm{bc}$ \\
\hline $5 \% \mathrm{Ca}\left(\mathrm{H}_{2} \mathrm{PO}_{4}\right)_{2}$ & $18.15 \mathrm{ef}$ & $19.83 \mathrm{ef}$ & $16.55 \mathrm{fg}$ & $18.18 \mathrm{fg}$ & $20.04 f g$ & $21.78 \mathrm{hi}$ & $21.31 \mathrm{de}$ & $21.05 \mathrm{gh}$ \\
\hline $1 \% \mathrm{H}_{2} \mathrm{O}_{2}$ & $20.28 b$ & $21.83 b$ & $21.54 \mathrm{ab}$ & $21.22 \mathrm{ab}$ & $23.57 \mathrm{~b}$ & $25.51 \mathrm{~b}$ & $24.34 \mathrm{ab}$ & $24.47 \mathrm{~b}$ \\
\hline $3 \% \mathrm{H}_{2} \mathrm{O}_{2}$ & $19.51 b c$ & $21.13 b-d$ & $20.05 b-d$ & $20.23 b c$ & $22.02 \mathrm{~cd}$ & $23.79 d-f$ & $22.12 \mathrm{~cd}$ & $22.65 d-f$ \\
\hline $5 \% \mathrm{H}_{2} \mathrm{O}_{2}$ & $18.38 \mathrm{~d}-\mathrm{f}$ & $20.34 d-f$ & $18.33 \mathrm{de}$ & $19.02 d-f$ & $20.94 d-f$ & $22.73 f-h$ & $20.26 \mathrm{ef}$ & $21.31 \mathrm{gh}$ \\
\hline Hydropriming & $17.93 \mathrm{f}$ & $19.53 \mathrm{fg}$ & $17.38 \mathrm{ef}$ & $18.28 \mathrm{e}-\mathrm{g}$ & $22.72 b c$ & $24.55 b-d$ & $22.99 b c$ & $23.42 \mathrm{~cd}$ \\
\hline Non-priming & $9.96 \mathrm{j}$ & $11.46 \mathrm{j}$ & $11.58 \mathrm{j}$ & $11.00 \mathrm{k}$ & $14.72 \mathrm{j}$ & $16.22 \mathrm{k}$ & $15.93 \mathrm{~h}$ & $15.62 \mathrm{k}$ \\
\hline $\mathrm{S}_{\mathrm{x}}^{-}$ & 0.328 & 0.331 & 0.569 & 0.345 & 0.411 & 0.354 & 0.435 & 0.294 \\
\hline Level of signific. & $* *$ & $* *$ & $* *$ & $* *$ & $* *$ & $* *$ & $* *$ & $* *$ \\
\hline $\mathrm{CV}(\%)$ & 3.24 & 2.97 & 5.83 & 3.34 & 3.40 & 2.70 & 3.54 & 2.35 \\
\hline
\end{tabular}

Figures with similar letter(s) or without letter within the column do not differ significantly whereas figures with dissimilar letter(s) differ significantly at 0.05 level of probability by DMRT, $\quad * *=$ Significant at 0.01 level of probability. 
Table 3. Effect of osmopriming treatment on mean germination time of maize seed during 2008.

\begin{tabular}{|c|c|c|c|c|c|c|c|c|}
\hline \multirow{3}{*}{ Treatments } & \multicolumn{8}{|c|}{ Mean germination time (day) } \\
\hline & \multicolumn{4}{|c|}{$30 \%$ moisture } & \multicolumn{4}{|c|}{$60 \%$ moisture } \\
\hline & \begin{tabular}{|l} 
Trial-1(March) \\
\end{tabular} & Trial-2 (Oct.) & Trial-3(Nov.) & Mean & Trial-1(March) & Trial-2 (Oct.) & Trial-3(Nov.) & Mean \\
\hline $1 \% \mathrm{Na}=\mathrm{SO}_{4}$ & $3.44 \mathrm{ef}$ & $3.42 \mathrm{e}-\mathrm{g}$ & $4.19 b$ & $3.68 \mathrm{de}$ & $3.38 \mathrm{~d}-\mathrm{f}$ & $3.41 \mathrm{e}-\mathrm{h}$ & $3.80 \mathrm{c}-\mathrm{f}$ & $3.53 \mathrm{e}-\mathrm{h}$ \\
\hline $3 \% \mathrm{Na}_{2} \mathrm{SO}_{4}$ & $3.65 b-d$ & $3.62 \mathrm{~b}-\mathrm{d}$ & $4.55 \mathrm{a}$ & $3.94 b$ & $3.53 b-d$ & $3.51 \mathrm{c}-\mathrm{e}$ & $3.91 b-d$ & $3.66 \mathrm{bc}$ \\
\hline $5 \% \mathrm{Na}_{2} \mathrm{SO}_{4}$ & $3.71 b c$ & $3.67 \mathrm{bc}$ & $4.56 \mathrm{a}$ & $3.98 b$ & $3.58 \mathrm{bc}$ & $3.59 \mathrm{bc}$ & $3.95 b c$ & $3.71 \mathrm{~b}$ \\
\hline $1 \% \mathrm{~K}_{2} \mathrm{HPO}_{4}$ & $3.47 \mathrm{~d}-\mathrm{f}$ & $3.48 \mathrm{e}-\mathrm{g}$ & $3.79 \mathrm{c}-\mathrm{g}$ & $3.58 \mathrm{~d}-\mathrm{g}$ & $3.39 \mathrm{~d}-\mathrm{f}$ & $3.38 \mathrm{~g}-\mathrm{I}$ & $3.75 \mathrm{~d}-\mathrm{g}$ & $3.51 \mathrm{f}-\mathrm{h}$ \\
\hline $3 \% \mathrm{~K}_{2} \mathrm{HPO}_{4}$ & $3.54 \mathrm{c}-\mathrm{e}$ & $3.53 \mathrm{c}-\mathrm{f}$ & $3.96 \mathrm{~b}-\mathrm{e}$ & $3.68 \mathrm{~cd}$ & $3.54 b-d$ & $3.52 \mathrm{~cd}$ & $3.87 \mathrm{~b}-\mathrm{e}$ & $3.64 b-d$ \\
\hline $5 \% \mathrm{~K}_{2} \mathrm{HPO}_{4}$ & $3.73 b$ & $3.69 b$ & $4.07 b c$ & $3.83 \mathrm{bc}$ & $3.47 \mathrm{c}-\mathrm{e}$ & $3.47 \mathrm{~d}-\mathrm{g}$ & $4.01 \mathrm{~b}$ & $3.65 b-d$ \\
\hline $1 \% \mathrm{ZnSO}_{4}$ & $3.44 \mathrm{ef}$ & $3.40 \mathrm{fg}$ & $3.94 b-f$ & 3.60d-f & $3.45 c-f$ & $3.43 \mathrm{~d}-\mathrm{h}$ & $3.83 b-f$ & $3.57 \mathrm{c}-\mathrm{f}$ \\
\hline $3 \% \mathrm{ZnSO}_{4}$ & $3.32 \mathrm{f}$ & $3.33 \mathrm{~g}$ & $3.54 \mathrm{~g}$ & $3.40 \mathrm{~g}$ & $3.31 \mathrm{f}$ & $3.30 \mathrm{I}$ & $3.57 \mathrm{~h}$ & $3.39 \mathrm{i}$ \\
\hline $5 \% \mathrm{ZnSO}_{4}$ & $3.48 \mathrm{~d}-\mathrm{f}$ & $3.49 \mathrm{~d}-\mathrm{f}$ & $4.14 b$ & $3.71 \mathrm{~cd}$ & $3.42 \mathrm{c}-\mathrm{f}$ & $3.47 \mathrm{~d}-\mathrm{g}$ & $3.84 b-f$ & $3.57 \mathrm{c}-\mathrm{f}$ \\
\hline $1 \% \mathrm{Ca}\left(\mathrm{H}_{2} \mathrm{PO}_{4}\right)_{2}$ & $3.47 \mathrm{ef}$ & $3.46 \mathrm{e}-\mathrm{g}$ & $3.79 \mathrm{c}-\mathrm{g}$ & $3.57 \mathrm{~d}-\mathrm{g}$ & $3.43 \mathrm{c}-\mathrm{f}$ & $3.45 \mathrm{~d}-\mathrm{h}$ & $3.76 \mathrm{~d}-\mathrm{g}$ & $3.55 \mathrm{~d}-\mathrm{g}$ \\
\hline $3 \% \mathrm{Ca}\left(\mathrm{H}_{2} \mathrm{PO}_{4}\right)_{2}$ & $3.42 \mathrm{ef}$ & $3.40 \mathrm{fg}$ & $3.64 d-g$ & $3.49 \mathrm{e}-\mathrm{g}$ & $3.37 \mathrm{~d}-\mathrm{f}$ & $3.36 \mathrm{hi}$ & $3.71 \mathrm{e}-\mathrm{h}$ & $3.48 \mathrm{hi}$ \\
\hline $5 \% \mathrm{Ca}\left(\mathrm{H}_{2} \mathrm{PO}_{4}\right)_{2}$ & $3.55 \mathrm{c}-\mathrm{e}$ & $3.57 \mathrm{~b}-\mathrm{e}$ & $3.98 \mathrm{~b}-\mathrm{d}$ & $3.69 \mathrm{~cd}$ & $3.50 \mathrm{~b}-\mathrm{e}$ & $3.45 \mathrm{~d}-\mathrm{h}$ & $3.81 \mathrm{c}-\mathrm{f}$ & $3.59 \mathrm{c}-\mathrm{f}$ \\
\hline $1 \% \mathrm{H}_{2} \mathrm{O}_{2}$ & $3.40 \mathrm{ef}$ & $3.39 \mathrm{fg}$ & $3.60 \mathrm{fg}$ & $3.46 \mathrm{fg}$ & $3.36 \mathrm{ef}$ & $3.35 \mathrm{hi}$ & $3.61 \mathrm{gh}$ & $3.44 \mathrm{~g}-\mathrm{i}$ \\
\hline $3 \% \mathrm{H}_{2} \mathrm{O}_{2}$ & $3.44 \mathrm{ef}$ & $3.41 \mathrm{fg}$ & $3.67 \mathrm{~d}-\mathrm{g}$ & $3.50 \mathrm{e}-\mathrm{g}$ & $3.51 \mathrm{~b}-\mathrm{e}$ & $3.49 \mathrm{~d}-\mathrm{f}$ & $3.85 b-f$ & $3.62 \mathrm{~b}-\mathrm{e}$ \\
\hline $5 \% \mathrm{H}_{2} \mathrm{O}_{2}$ & $3.49 \mathrm{~d}-\mathrm{f}$ & $3.44 \mathrm{e}-\mathrm{g}$ & $3.78 \mathrm{c}-\mathrm{g}$ & $3.57 \mathrm{~d}-\mathrm{g}$ & $3.65 b$ & $3.64 \mathrm{~b}$ & $3.87 \mathrm{~b}-\mathrm{e}$ & $3.72 b$ \\
\hline Hydropriming & $3.38 \mathrm{ef}$ & $3.42 \mathrm{fg}$ & $3.63 \mathrm{e}-\mathrm{g}$ & $3.47 \mathrm{fg}$ & $3.36 \mathrm{ef}$ & $3.39 \mathrm{f}-\mathrm{I}$ & $3.68 \mathrm{f}-\mathrm{h}$ & $3.46 \mathrm{f}-\mathrm{h}$ \\
\hline Non-priming & $4.47 \mathrm{a}$ & $4.41 \mathrm{a}$ & $4.73 a$ & $4.54 \mathrm{a}$ & $4.50 \mathrm{a}$ & $4.45 \mathrm{a}$ & $4.59 \mathrm{a}$ & $4.52 \mathrm{a}$ \\
\hline $\mathrm{S}_{\mathrm{x}}^{-}$ & 0.054 & 0.044 & 0.103 & 0.051 & 0.048 & 0.031 & 0.054 & 0.031 \\
\hline Level of signific. & $* *$ & $* *$ & $* *$ & $* *$ & $* *$ & $* *$ & $* *$ & $* *$ \\
\hline $\mathrm{CV}(\%)$ & 2.63 & 2.16 & 4.47 & 2.41 & $2.35 \%$ & 1.54 & $2.42 \%$ & 1.49 \\
\hline
\end{tabular}

Figures with similar letter(s) or without letter within the column do not differ significantly whereas figures with dissimilar letter(s) differ significantly at 0.05 level of probability by DMRT, $* *=$ Significant at 0.01 level of probability. 


\section{Discussion}

Good seedling establishment is very important for successful maize production because the crop has no capacity to adjust sub-optimal stand by tillering. Seed priming could be used as a viable technology to improve seedling establishment. Rapid and uniform emergence and yield improvement have been achieved by seed priming in vegetables (Bruggink et al., 1999) and in some field crops (Giri and Schillinger, 2003; Murungu et al., 2004). Osmopriming helps improving germination in many crop seeds. Bailly et al. (2000); Kattimani et al. (1999); Guzman and Olave (2006) observed similar results in maize. The results of the experiment showed that germination percentage, germination index, and mean germination time varied significantly due to different osmopriming treatments. The highest emergence of maize seed was noticed with osmopriming by $3 \%$ $\mathrm{ZnSO}_{4}$. The emergence performance of osmoprimed seeds was also better than hydroprimed seed. Some metabolic and physiological changes occur in the primed seeds which are helpful for faster growth of embryo. Moreover, seed priming contribute to earlier DNA replication, increased RNA and protein synthesis, greater ATP availability, repair of deteriorated seed parts and reduced leakage of metabolites. As a result, osmoprimed seed increase emergence, reduce seedling germination time and improve stand establishment of maize. This result is at par with the observation of Salehzadi et al. (2009); Golezani et al. (2008) and Farooq et al. (2005). The result indicated that better seed emergence could be achieved by osmopriming with $3 \% \quad \mathrm{ZnSO}_{4}$ solution. Osmoprimed seed of maize also gave better performance in seed emergence than non-primed seed either at optimum condition or even at stressful environment. So, osmoprimed seed could be used as a tool to improve seedling emergence and better seedling establishment.

\section{Conclusion}

Osmopriming of maize seed with $3 \% \mathrm{ZnSO}_{4}$ solution gave best emergence under laboratory condition.

\section{References}

AOSA (Association of Official Seed Analysts).1983. Seed Vigor Testing Handbook. Contribution No. 32 to the handbook on Seed Testing.

Bailly, C., A. Benamar, F. Corbineau and D. Come. 2000. Antioxidant systems in sunflower (Helianthus annuus L.) seeds as affected by priming. Seed Sci. Res. 10: 35-42.

Basra, S.M.A., M. Farooq, R. Tabsassam and N. Ahmad. 2005. Physiological and biochemical aspects of pre-sowing seed treatments in fine rice (Oryza sativa L.). Seed Sci. Technol. 33:

Bruggink, G.T., J.J.J. Ooms, and P. Van der Toorn. 1999. Induction of longevity in primed seeds. Seed Sci. Res. 9: 49-53. 
Farooq, M., S.M.A. Basra, K. Hafeez and N. Ahmad. 2005. Thermal hardening: A new seed vigour enhancement tool in rice. Acta Bot. Sin. 47: 187-193.

Finch-Savage, W.E., K.C. Dent and L.J. Clark. 2004. Soak conditions and temperature following sowing influence the response of maize (Zea mays L.) seeds to on-farm priming (pre-sowing seed soak). Field Crops Res. 90(2/3): 361-374.

Ghiyasi, M., A.S. Abbasi, M. Tajbakhsh, R. Amirnia and H. Salehzade. 2008. Effect of osmopriming with poly ethylene glycol $8000\left(\mathrm{PEG}_{8000}\right)$ on germination and seedling growth of wheat (Triticum aestivum L.) seeds under salt stress. Res. J. Biol. Sci. 3(10): 1249-1251.

Giri, G.S. and W.F. Schillinger. 2003. Seed priming winter wheat for germination, emergence and yield. Crop Sci. 43: 2135-2141.

Golezani, K.G., A.A. Aliloo, M. Valizadeh and M. Moghaddam. 2008. Effects of different priming techniques on seed invigoration and seedling establishment of lentil (Lens culinaris Medik). J. Food Agric. Environ. 6(2): 222-226.

Gomez K.A. and A.A. Gomez. 1984. Statistical Procedures for Agriculture Research. Intl. Rice. Res. Inst., Philippines, Pp. 187-411.

Guzman, M. and J. Olave. 2006. Response of growth and biomass production of primed melon seed (Cucumis melo L. cv. Primal) germination to salinity level and N-forms in nursery. J. Food Agric. Environ. 4: 163-165.

Harris, D., A.K. Pathan, P. Gothkar, A. Joshi, W. Chivasa and P. Nyamudeza. 2001. Onfarm seed priming: using participatory methods to revive and refine a key technology. Agril. Systems 69:151-164.

Heydecker, W. J., J. Higgins and K. Gulliver. 1973. Accelerated germination by osmotic seed treatment. Nature 246: 42-46.

Kattimani, K.N., Y.N. Reddy and B. Rajeswar Rao. 1999. Effect of pre-sowing seed treatment on germination, seedling emergence, seedling vigour and root yield of Ashwagandha (Withania somnifera Daunal.). Seed Sci. Technol. 27: 483-488.

Lee, S.S. and H.H. Kim. 1999. Morphological change, sugar content, $\alpha$-amylase activity of rice seeds under various priming conditions. Korean J. Crop Sci. 44: 138-142.

Murungu, F.S., C. Chiduza, P. Nyamugafata, L.J. Clark, W.R. Whalley and W.E. FinchSavage. 2004. Effects of on-farm seed priming on consecutive daily sowing occasions on the emergence and growth of maize in semi-arid Zimbabwe. Field Crops Res. 89(1): 49-57.

Ozbingol N., F. Corbineau and D. Come. 1998. Response of tomato seeds to osmoconditioning as related to temperature and oxygen. Seed Sci. Res. 8: 377-384.

Salehzade, H., M.I.Shishvan, M.Ghiyasi, F. Forouzin and A. Siyahjani. 2009. Effect of seed priming on germination and seedling growth of wheat (Triticum aestivum L.). Res. J. Biol. Sci. 4(5): 629-631.

Scott, S. L., R.A. Jones and W.A. Williams. 1984. Review of data analysis methods for seed germination. Crop Sci. 24:1192-1198. 